\title{
Modelling the immune response to cancer: an individual-based approach accounting for the difference in movement between inactive and activated T cells
}

\author{
Fiona R Macfarlane • Tommaso Lorenzi • \\ Mark A J Chaplain
}

Received: date / Accepted: date

\begin{abstract}
A growing body of experimental evidence indicates that immune cells move in an unrestricted search pattern if they are in the pre-activated state, while they tend to stay within a more restricted area upon activation induced by the presence of tumour antigens. This change in movement is not often considered in the existing mathematical models of the interactions between immune cells and cancer cells. With the aim to fill such a gap in the existing literature, in this work we present a spatially structured individual-based model of tumour-immune competition that takes explicitly into account the difference in movement between inactive and activated immune cells. In our model, a Lévy walk is used to capture the movement of inactive immune cells, whereas Brownian motion is used to describe the movement of antigen-activated immune cells. The effects of activation of immune cells, the proliferation of cancer cells and the immune destruction of cancer cells are also modelled. We illustrate the ability of our model to reproduce qualitatively the spatial trajectories of immune cells observed in experimental data of single cell tracking. Computational simulations of our model further clarify the conditions for the onset of a successful immune action against cancer cells and suggest possible targets to improve the efficacy of cancer immunotherapy. Overall, our theoretical work highlights the importance of taking into account spatial interactions when modelling the immune response to cancer cells.
\end{abstract}

Keywords Cancer-Immune Competition · Spatial Movement · Individual-Based Models · Lévy Walk · Brownian Motion

\section{Introduction}

Recent advances in technology have allowed for a deeper understanding of the individual interactions occurring between cancer cells and cells of the human im-

F R Macfarlane funded by the Engineering and Physical Sciences Research Council (EPSRC)

F R Macfarlane

School of Mathematics and Statistics, University of St Andrews, St Andrews, KY16 9SS

Tel.: +44 1334463766

E-mail: frm3@st-andrews.ac.uk 
mune system, and how these could be exploited using immunotherapies (Basu et al, 2016; Boissonnas et al, 2007; Engelhardt et al, 2012; Halle et al, 2016; Pitt et al, 2016; Tan et al, 2015).

The immune system is made up of two parts: the innate response and the adaptive immune response. Cells of the innate immune response, such as natural killer (NK) cells, can cause limited damage to a solid tumour resulting in tumour cell debris in the microenvironment. This debris contains tumour antigens (i.e. smaller proteins produced by the tumour) which can be ingested by nearby innate dendritic cells (DCs) (Messerschmidt et al, 2016). The DCs can then activate adaptive cytotoxic T lymphocytes (CTLs) through antigen presentation, which is a key step in determining whether the immune system will be capable of removing a tumour (Boissonnas et al, 2007; Engelhardt et al, 2012). Once the activated CTLs have reached the site of the cancer cells, they can cause cancer cell death via apoptosis through direct interaction or release of chemicals (Hersey and Zhang, 2001; Modiano and Bellgrau, 2016).

Immunotherapies based on $\mathrm{T}$ lymphocytes have been widely researched recently, with the aim to enhance the response of $\mathrm{T}$ lymphocytes to solid tumours (Andersen et al, 2016; Frigault and Maus, 2016; Gross and Eshhar, 2016; Ikeda, 2016). Adoptive $\mathrm{T}$ cell therapies rely on $\mathrm{T}$ lymphocytes from the patient, or from donor patients, to be modified and then (re)injected into the patient. The lymphocytes are removed from the body and then undergo incubation with cytokines to allow for expansion ex vivo. They are then transferred back into the patient with the aim that they are able to specifically target cancer cells (Gross and Eshhar, 2016). In a similar method dendritic cells can be removed from the body and expanded or activated using tumour antigens ex vivo, promoting an enhanced response of $\mathrm{T}$ lymphocytes once reinjected into the patient (Carreno et al, 2015; Prue et al, 2015; Schreibelt et al, 2016; Tel et al, 2013; Weinberg, 2007a; Wilgenhof et al, 2016, 2011).

The use of mathematical modelling to capture the interactions between the immune system and cancer has been widely examined over the past few decades. The majority of the models use ordinary differential equations (ODEs) or integrodifferential equations (IDEs) to describe cellular level dynamics, with many of them focusing on tumour cells interacting with cytotoxic T lymphocytes (Bellomo and Delitala, 2008; Bunimovich-Mendrazitsky et al, 2008; Cattani et al, 2010; Delitala and Lorenzi, 2013; Frascoli et al, 2014; Kolev, 2003; Kuznetsov and Knott, 2001; Kuznetsov et al, 1994; Lin Erickson et al, 2009; Lorenzi et al, 2015; Takayanagi and Ohuchi, 2001; Wilkie and Hahnfeldt, 2013). From these non-spatial models the effect of immune evasion by the cancer cells has been well described, with the parameter values of the models being derived either from experimental data or estimated logically. Key dynamics can be confirmed or expanded in a wider range of biologically relevant situations by developing these ODE and IDE models into partial differential equation (PDE) models through the inclusion of spatial movement (e.g. random motility, chemotaxis) of the cells (Al-Tameemi et al, 2012; d'Onofrio and Ciancio, 2011; Matzavinos et al, 2004). Models of this type allow for a deeper understanding of the immune evasion processes and have highlighted situations of cancer dormancy, where a small tumour continues to exist but is maintained at a restricted size by the immune system. They can also shed light on immunotherapies and cancer vaccination protocols (Joshi et al, 2009). Differential equation models are useful and, in addition to providing insight through simu- 
lation, are amenable to mathematical analysis to achieve conclusions concerning structural stability under parameter changes. However, the differential formalism poses some limitation to the level of biological detail which can be incorporated in these models. By using computational models such as cellular automata (CA) and individual-based models (IBMs) a wider spectrum of biological phenomena can be translated into mathematical terms and described. These models can be posed on a spatial domain (e.g. a grid), and a set of rules can be given to each cell with certain probabilities to achieve a more detailed description of cancerimmune competition (Chowdhury et al, 1991; Christophe et al, 2015; Hu et al, 2012; Pappalardo et al, 2008).

Advances in imaging techniques have allowed individual cell tracking to become a reality, and through this a key aspect of immune cell motion has been discovered, i.e. CTLs move in an unrestricted search pattern if they are in the pre-activated state. This allows them to move larger distances more quickly. However, once activated via presentation of a tumour antigen, CTLs adopt a more restricted movement, allowing them to stay within a confined area (Boissonnas et al, 2007). DCs have been shown to have similar properties and their movement becomes restricted after obtaining a tumour antigen (Engelhardt et al, 2012). This change in the type of movement is not often considered in the existing mathematical models of the interactions between immune cells and cancer cells. With the aim to fill such a gap in the existing literature, in this paper we present a spatially structured individual-based model of tumour-immune competition that explicitly takes into account the difference in movement between inactive and activated immune cells. In our model, a Lévy walk is used to model the movement of inactivated immune cells, whereas Brownian motion is used to describe the movement of antigen-activated immune cells. Additionally, the effects of activation of immune cells, proliferation of cancer cells and immune destruction of tumour cells are incorporated in the model.

The remainder of the paper is organised as follows. In Section 2, we outline the mathematical model and describe how we parametrise the model using data from the existing literature. In Section 3, we present the main computational results that we obtain varying the model parameters to consider a number of biological scenarios. Finally, in Section 4 we discuss the implications of these results and present directions for future work.

\section{The mathematical model}

In our model three cell types are considered: cancer cells (from a solid tumour), dendritic cells and cytotoxic $\mathrm{T}$ lymphocytes. The total numbers of these cells will be denoted by $N_{T}, N_{D}$ and $N_{C}$, respectively. We describe the interactions occurring between the three cell types in an in vitro situation using an individualbased modelling approach.

Our IBM is posed on a $2 \mathrm{D}$ spatial grid of spacing $\Delta_{x}$ in the $x$ direction and $\Delta_{y}$ in the $y$ direction, with the constraint that only one cell of any type is allowed at each grid-site at any time-step of duration $\Delta_{t}$. 

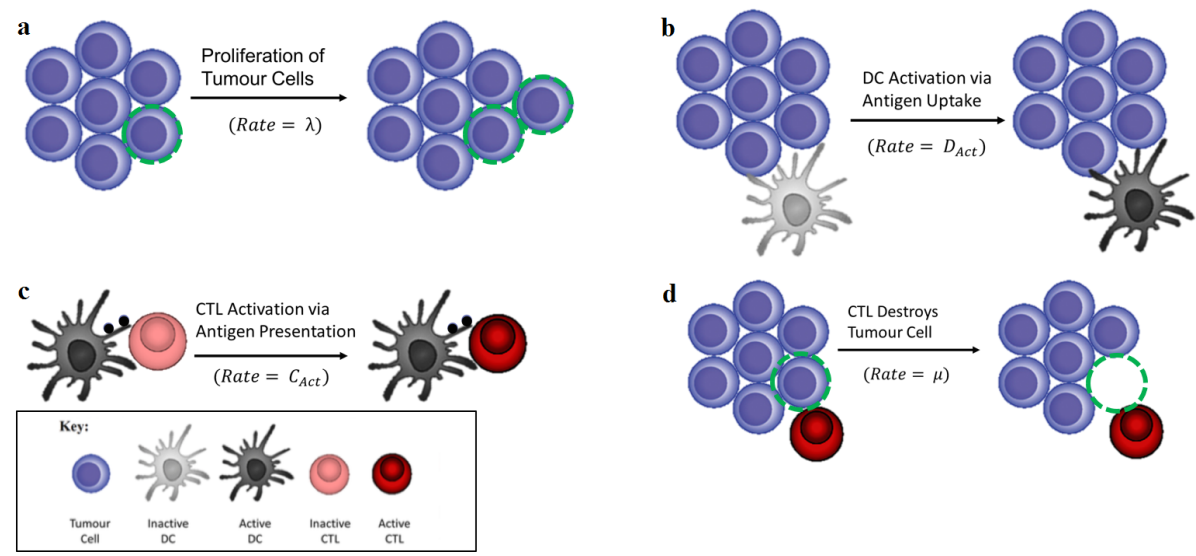

d

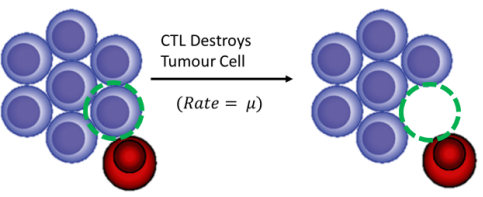

Fig. 1 Mechanisms and processes included in the model. Three different cell types and five different cell states are considered in the model (Key subpanel). (a) Tumour cells can proliferate if a neighbouring position is free at rate $\lambda$. (b) Dendritic cells (DCs) can become activated by uptake of antigen from the tumour upon contact at rate $D_{A c t}$. (c) Activated DCs can present the antigen to inactive cytotoxic $\mathrm{T}$ lymphocytes (CTLs) upon contact allowing them to become activated at rate $C_{A c t}$. (d) Activated CTLs can remove tumour cells upon contact at rate $\mu$

\subsection{Proliferation of tumour cells}

We allow the (solid) tumour to grow through cancer cell proliferation. A cancer cell divides at rate $\lambda$ into two daughter cells of which one occupies the position of the mother cell while the other is positioned at an unoccupied neighbouring grid-site, Fig.1a. This ensures that only cancer cells with free grid-sites in their neighbourhood can proliferate.

In early stages of tumourigenesis, cancer cells must stay close to the original site of the tumour (Hanahan and Weinberg, 2011). Once the tumour has developed and is large enough, single cells can leave the tumour mass and travel to other sites of the body (Weinberg, 2007b). In our model, we consider a case where the tumour is at an early stage of its development and not significantly large enough to initiate this process (invasion and metastasis), and so we do not consider cancer cell movement. Moreover, self-induced cell death of the cancer cells is omitted from the model, a factor which can be explained by telomeric shortening. In fact, the process that leads human cells to induce their own cell death depends on the telomeres at the end of chromosomes shortening. Once the telomeres shorten significantly the cell can no longer replicate and will initiate apoptosis. Most cancer cells express high levels of telomerase, an enzyme that prevents the telomeres shortening allowing for infinite survival of cells (Shay et al, 2001).

\subsection{Movement of inactive immune cells}

We assume that initially both types of immune cells, CTLs and DCs, are in an inactive state and randomly distributed throughout the domain. Boissonnas et al (2007) used individual cell tracking to study the movement of CTLs in the presence 

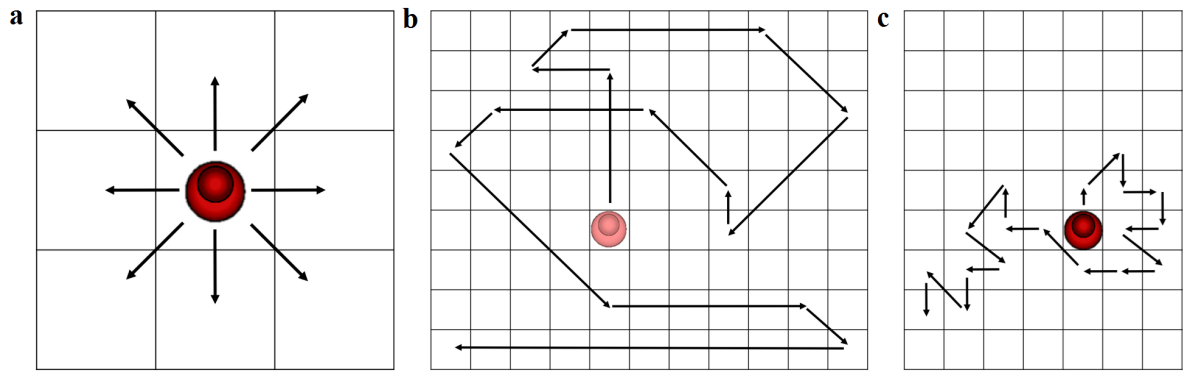

Fig. 2 Cell neighbourhood and mechanisms of immune cell motion. (a) At each time-step, immune cells can move to one of the eight neighbouring grid-sites provided it is unoccupied. (b) Inactive immune cells move via a Lévy walk and the probability of moving in a given direction for $s$ time-steps is taken from the Lévy distribution given by Eq.(1). (c) Activated immune cells move via Brownian motion and the probability of moving to neighbouring grid-sites is the same at each time-step

and absence of a tumour antigen, and verified a change in the type of movement between these situations. In the case where no antigen was present, the cells moved actively in a search like pattern. On the other hand, when the tumour antigen was present, the cells switched to a form of movement that leads them to cover a more restricted portion of space during the same time interval. This corresponds to situations where the immune cells will either be inactive or activated by the tumour antigen. Engelhardt et al (2012) discovered similar results when studying the movement of dendritic cells in response to a solid tumour, where the change in movement was linked to antigen activation. In order to incorporate this into our model, we choose two forms of random walk to describe immune cell movement: we let inactive cells move via a Lévy walk mechanism and activated cells move via Brownian motion. At each time-step, cells move to an unoccupied neighbouring grid-site, Fig.2a. If a cell is using a Lévy walk, the direction of movement is initially chosen randomly (i.e. with equal probability) from one of the eight directions shown in Fig.2a. Then the number of time steps $s$ that the cell persists in this direction is chosen from the Lévy distribution given by Eq.(1). If the number of steps is one (i.e. $s=1$ ), then the process repeats at the next time-step, otherwise the cell will keep moving in the initially chosen direction until all steps have been taken. Once the step-number is zero, the process begins again. The mechanism follows the rule that cells cannot move to an occupied grid-site or a grid-site outside the boundary of the domain. The Lévy distribution is defined as

$$
\mathrm{P}(s)=s^{-(\alpha+1)}
$$

where $s$ is the number of time-steps the cell will move in a given direction and $\alpha$ is the walk exponent (see Harris et al (2012) for full details). This means that cells whose motion is governed by a Lévy walk have a larger probability of moving in a given direction for a larger number of time-steps than a cell undergoing Brownian motion, Fig.2b. 
2.3 Movement of activated immune cells

Once activated (see the following subsection), both types of immune cell alter their movement from the Lévy walk mechanism to a more spatially restricted mechanism of Brownian motion. Therefore, activated cells will have equal probability, at each time-step, to move to one of the eight neighbouring grid-sites, Fig.2c. If the desired grid-site is occupied or outside the domain the cell will not move to the new site.

As we are considering an in vitro system, we assume that there will be no influx or escape of immune cells into or from the domain, respectively. Using reflective boundary conditions we ensure that cells cannot leave the domain. On first approximation, we assume the total numbers of DCs and CTLs to remain constant over time, that is, we omit the effects of proliferation and natural decay of both CTLs and DCs.

\subsection{Activation of immune cells}

Inactive DCs can become activated by contact with the cancer cells. In the model, we assume that when DCs are at a neighbouring grid-site to a cancer cell, the two cells can interact and this can result in activation of the DCs at rate $D_{A c t}$, Fig.1b.

Inactive CTLs can become activated via interactions with activated DCs. If activated DCs and inactive CTLs are at neighbouring grid-sites they can interact and this may result in the activation of the CTLs with rate $C_{A c t}$, Fig.1c. Once a DC has activated a CTL it can go on to activate other cells as we do not consider any deactivation or exhaustion processes.

\subsection{Destruction of tumour cells}

Activated CTLs can interact with tumour cells if they are at neighbouring gridsites. The cancer cell can be removed upon interaction with a CTL at rate $\mu$, Fig.1d. Once a CTL has interacted with a cancer cell it can continue to seek out and remove other cancer cells, since deactivation and exhaustion processes are not considered here.

\section{Simulation results}

3.1 Model parametrisation and numerical set up

The mathematical model is parametrised using parameter values obtained from published biological data wherever possible. The full list of parameters and related references can be found in Table 2 in the Appendix.

To fix the size of the grid spacing, we consider the average diameters of all three cell types, where melanoma cells are chosen as the cancer cells (see Table 1). To reduce biological complexity to its essence, we assume each grid-site to be 10 $\mu m$ apart in both the $x$ and $y$ direction. Therefore we set

$$
\Delta_{x}=10 \mu \mathrm{m} \quad \text { and } \quad \Delta_{y}=10 \mu \mathrm{m} .
$$


Table 1 Average diameter of cell types considered

\begin{tabular}{lll}
\hline Cell Type & Average Diameter & Reference \\
\hline Cytotoxic T lymphocyte & $9-16 \mu \mathrm{m}$ & (Rozenberg, 2011) \\
Dendritic cell & $\sim 7 \mu \mathrm{m}$ & (Goya et al, 2008) \\
Melanoma cell & $\sim 12.5 \mu \mathrm{m}$ & (Christophe et al, 2015) \\
\hline
\end{tabular}

Using 100 grid points in both the $x$ and $y$ directions, the 2D spatial domain is therefore of size $1 \mathrm{~mm}^{2}$, representing a $1 \mathrm{~mm}^{2}$ in vitro sample.

To determine the time-step length $\Delta_{t}$ we consider the speed of the immune cells. The average speed of both inactive and activated CTLs has been found to be approximately $10 \pm 5 \mu \mathrm{m} \mathrm{min}^{-1}$ (Boissonnas et al, 2007). Using an average cell speed of $10 \mu \mathrm{m} \mathrm{min}^{-1}$ for both immune cell types we choose

$$
\Delta_{t}=1 \mathrm{~min}
$$

and so we let cells move one grid-step per time-step.

\subsubsection{Proliferation of tumour cells}

The proliferation rate of the cancer cells $\lambda$ was taken from (Christophe et al, 2015), where the authors calculated the estimated proliferation rate of a cancer cell using the average duplication time of melanoma cells. In particular, we choose the proliferation rate of our cancer cells to be

$$
\lambda=0.001 \min ^{-1} \text {. }
$$

At the beginning of simulations, the tumour mass is set up as a tightly packed circle of cancer cells. Therefore, only cancer cells on the periphery of the circle (circumference) will be eligible and able to proliferate.

\subsubsection{Movement of inactive immune cells}

As described previously, inactive cells will move using a Lévy walk mechanism taking their step length from the Lévy distribution given by Eq.(1). Harris et al (2012) studied the movement of CTLs in response to the pathogen Toxoplasma gondii in the brain of infected mice. Using cell imaging, the movement of the CTLs was tracked and matched to a Lévy walk where the step length was taken from a Lévy distribution with exponent

$$
\alpha=1.15 \text {. }
$$

\subsubsection{Activation of immune cells}

Dendritic cells can collect tumour antigens from the surface of tumour cells to become activated antigen presenting cells. Bianca et al (2012) found that the activation rate of DCs by a tumour cell was

$$
D_{\text {Act }}=0.07 \text { cells } \text { min }^{-1} \text {. }
$$


The activated DCs can then present the tumour antigen to the CTLs, activating them to seek out and destroy cells displaying the tumour antigen. From (Engelhardt et al, 2012) it can be calculated that it takes on average around 562 secs for a DC to activate a CTL through antigen presentation. This corresponds to an activation rate

$$
C_{\text {Act }} \approx 0.12 \text { cells } \text { min }^{-1} \text {. }
$$

\subsubsection{Movement of activated immune cells}

As described previously, activated immune cells will move using Brownian motion. At each time-step, these cells have an equal probability of moving to each of the neighbouring grid-sites, if they are unoccupied.

\subsubsection{Destruction of tumour cells}

Activated CTLs can interact and initiate apoptosis in cells that display the tumour antigen. Upon interaction with cancer cells, CTLs can initiate apoptosis in the cancer cell at rate $\mu$. Through studies using melanoma cells, Christophe et al (2015) found this rate to be

$$
\mu=0.038 \text { cells } \text { min }^{-1} \text {. }
$$

\subsection{Comparison with biological data of single CTL tracking}

To confirm that using a Lévy walk and a change to Brownian motion upon activation is an appropriate strategy for modelling immune cell motion, we attempted to replicate qualitatively the biological data of Boissonnas et al (2007). The experimental data considered CTL motion over time in the presence or absence of a tumour antigen. The data indicated that if the antigen is present the CTLs cover a more restricted area, whereas in the absence of the antigen CTLs move in a search type pattern exploring a wider portion of space over the same time interval. To reproduce in silico the experimental setting used in (Boissonnas et al, 2007), we considered 50 cells with their starting positions aligned and tracked each individual cell's trajectory over time in the case where cells movement was modelled by either Brownian motion, Fig.3c, or a Lévy walk, Fig.3d. The boundaries of the grid were reduced to match the biological results. Simulations were run over a time period chosen to be consistent with the time frame of the experimental data, ??? Online Resources 1-2 ???. Apart from cell motion no other mechanisms were included in this reduced version of the model. Our computational results are in good qualitative agreement with the experimental results obtained in the presence of a tumour antigen (compare the results in Fig.3c with the results in Fig.3a) and in the absence of the antigen (compare the results in Fig.3d with the results in Fig.3b). 


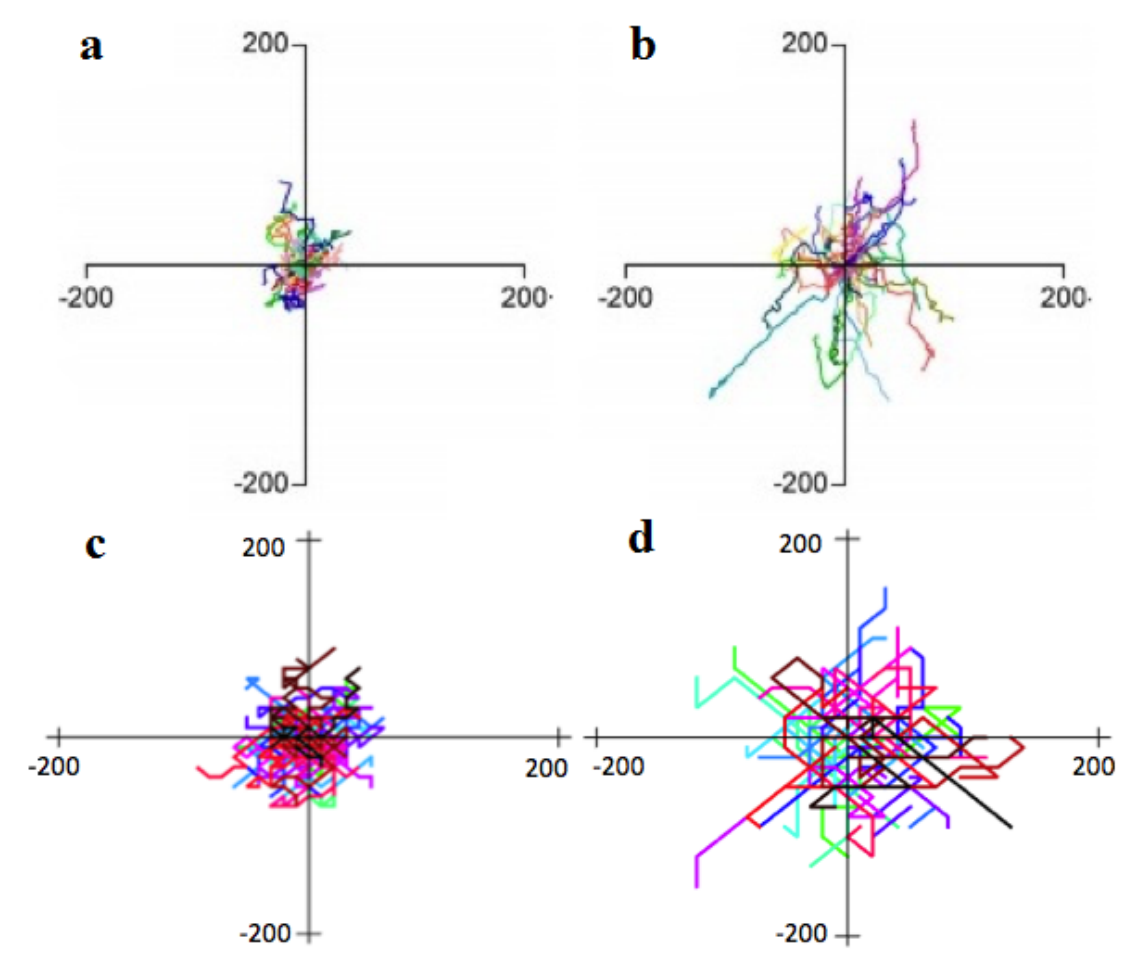

Fig. 3 Comparison with biological data of single CTL tracking. (a-b) Figures from (Boissonnas et al, 2007) displaying overlay of $50 \mathrm{CTL}$ tracks with aligned starting positions. (a) CTL motion in the presence of a tumour antigen. (b) CTL movement when there is no tumour antigen. (c-d) Computational results from our model showing overlay of 50 CTL tracks with aligned starting positions. (c) CTLs moving via Brownian motion. (d) CTLs moving via a Lévy walk

Fig.2B: Image 1, Image 3, used with permission, from (Boissonnas et al, 2007)

(c) 2007 A Boissonnas et al. Journal of Experimental Medicine. 204(2):345-356. doi: 10.1084/jem.20061890

3.3 Increasing the number of DCs can cause overcrowding and lead to longer tumour removal times

Adoptive cell transfer aims to increase the number of activated CTLs which are in the tumour microenvironment. Dendritic cell therapies have a similar approach increasing the number of activated DCs in order to promote the activation of CTLs. Bearing in mind these ideas, we can test the effect of varying the number of immune cells present in the system using our IBM. Simulations were run over a $72 \mathrm{hr}$ period, in line with the experiments of Christophe et al (2015), or until all of the tumour cells were removed from the system. All parameters remained at set values from Table 2 except for the total number of CTLs, $N_{C}$, and/or the total number of DCs, $N_{D}$, which were varied. Simulations were run with a range of 40 different values of $N_{C}, N_{D}$ or both $N_{C}$ and $N_{D}$, where total immune cell numbers ranged from 60 to 2400 cells. Heat maps were created to show the time evolution of the total tumour cell number for different immune cell numbers, left 
panels of Fig.4. Selected values of 60, 420, 1200 and 2400 immune cells were chosen to give a sample of the dynamics observed in each case. The time evolution of the total tumour cell number was plotted for each of these cases, right panels of Fig.4. Increasing the number of CTLs leads to a decrease in the time taken to remove the tumour, top panels of Fig.4. However for larger cell numbers, i.e. 1200 and 2400 CTLs, there is little difference in tumour removal time. Generally, increasing the DC number results into very little change in tumour removal time, middle panels of Fig.4. When considering the sample dynamics for this case, the cases with 1200 and 2400 DCs are associated with longer tumour removal times than the case with 420 DCs. Moreover, the largest case considered, i.e. 2400 DCs, leads to a longer tumour removal time than the smallest case considered, i.e. 60 DCs. Increasing both $N_{C}$ and $N_{D}$ causes an descrease in tumour removal time, bottom panels of Fig.4. As shown in Fig.9, similar dynamics are observed in the situation where the tumour was initially larger containing 1200 tumour cells instead of 400 tumour cells.

When carrying out simulations in the cases of increased immune cell numbers, we observe larger numbers of CTLs or DCs surrounding the tumour, which appear to cause overcrowding and limit the access of the activated CTLs. We can investigate this further by tracking the time evolution of the average distance of CTLs from the centre of the tumour. In the case where CTLs are varied only with either 1200 or 2400 cells we observe that in the latter case CTLs cannot get as close to the tumour centre, Fig.5a and Online Resources 3-4. When increasing the DC number only from 1200 to 2400 cells we see that CTLs are on average further away from the tumour centre and DCs appear to cause overcrowding, which pushes activated CTLs away from the tumour, Fig.5b and Online Resources 5-6. When both CTLs and DCs are increased from 1200 cells to 2400 cells, we again observe that the CTLs cannot get as close to the tumour centre in the latter case, Fig.5c and Online Resources 7-8.

3.4 The ratio between the killing rate of tumour cells by CTLs and the tumour cell proliferation rate is a crucial parameter in tumour removal

The rate at which a CTL can kill tumour cells has been previously shown to be a key parameter in tumour removal (Matzavinos et al, 2004). Therefore, we examine the effect of varying the rate at which a CTL can kill tumour cells, $\mu$, and we consider varying the tumour cell proliferation rate, $\lambda$. IBM simulations were again run over a $72 \mathrm{hr}$ period or until all of the tumour cells were removed from the system. All parameters remained at set values from Table 2 except for $\mu$ and $\lambda$. Simulations were run with a range of 40 values of $\mu, \lambda$ or both parameters. The values of these parameters were scaled ranging from a half to twenty times the original parameter values given in Table 2 . Heat maps were created to show the evolution of the total tumour cell number over time with varying values of $\mu$ and/or $\lambda$, left panels of Fig.6. Selected values of a half, one, ten and twenty times the original parameter values were chosen to give a sample of the dynamics in each case, right panels of Fig.6. Increasing $\mu$ leads to an decrease in tumour removal time, top panels of Fig.6. However, there is little difference in resulting dynamics when multiplying our original value of $\mu$ by ten or twenty. Increasing $\lambda$ leads to an increase in the tumour removal time, and larger values lead to a larger number of 

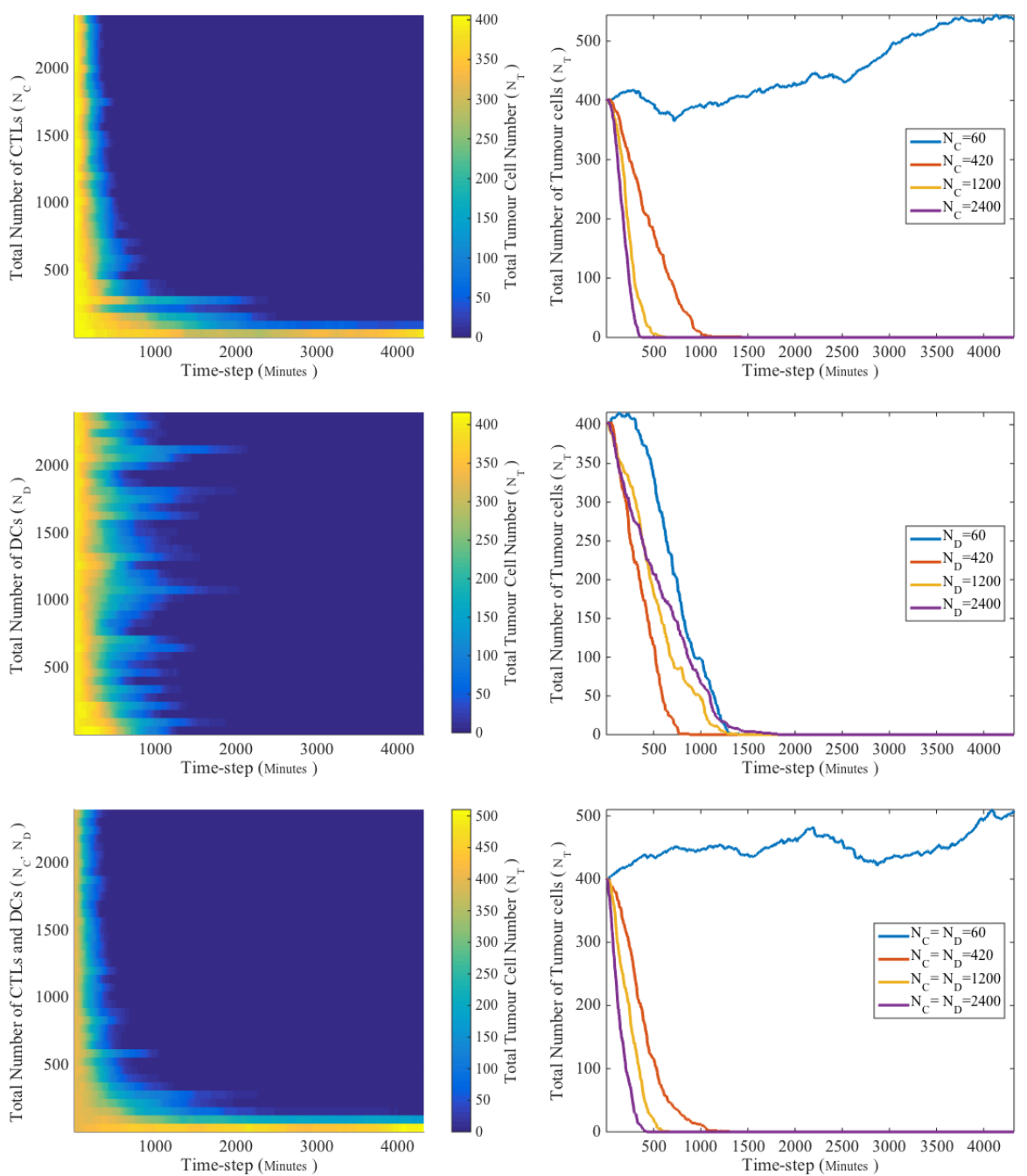

Fig. 4 Increasing the number of DCs can lead to longer tumour removal times. Heat maps showing the time evolution of the number of tumour cells for 40 different numbers of immune cells (left panels) and selected samples of the time evolution of the number of tumour cells for 4 different numbers of immune cells (right panels). In all cases under consideration, at the beginning of simulations the tumour contained 400 cells. Top panels: Increasing the CTL number $N_{C}$ we observe a general decrease in tumour removal time. Middle panels: Increasing the DC number $N_{D}$ above a certain threshold leads to longer tumour removal time. Bottom panels: Increasing both $N_{C}$ and $N_{D}$ causes a decrease in tumour removal time

tumour cells remaining after $72 \mathrm{hrs}$, middle panels of Fig.6. By increasing both $\lambda$ and $\mu$ we observe a general decrease in tumour removal time, slightly slower than the cases where only $\mu$ is altered, bottom panels of Fig.6. As shown in Fig.10, similar dynamics are observed in the case of an initially smaller tumour made up of 400 cells and an initially larger tumour made up of 1200 cells. 

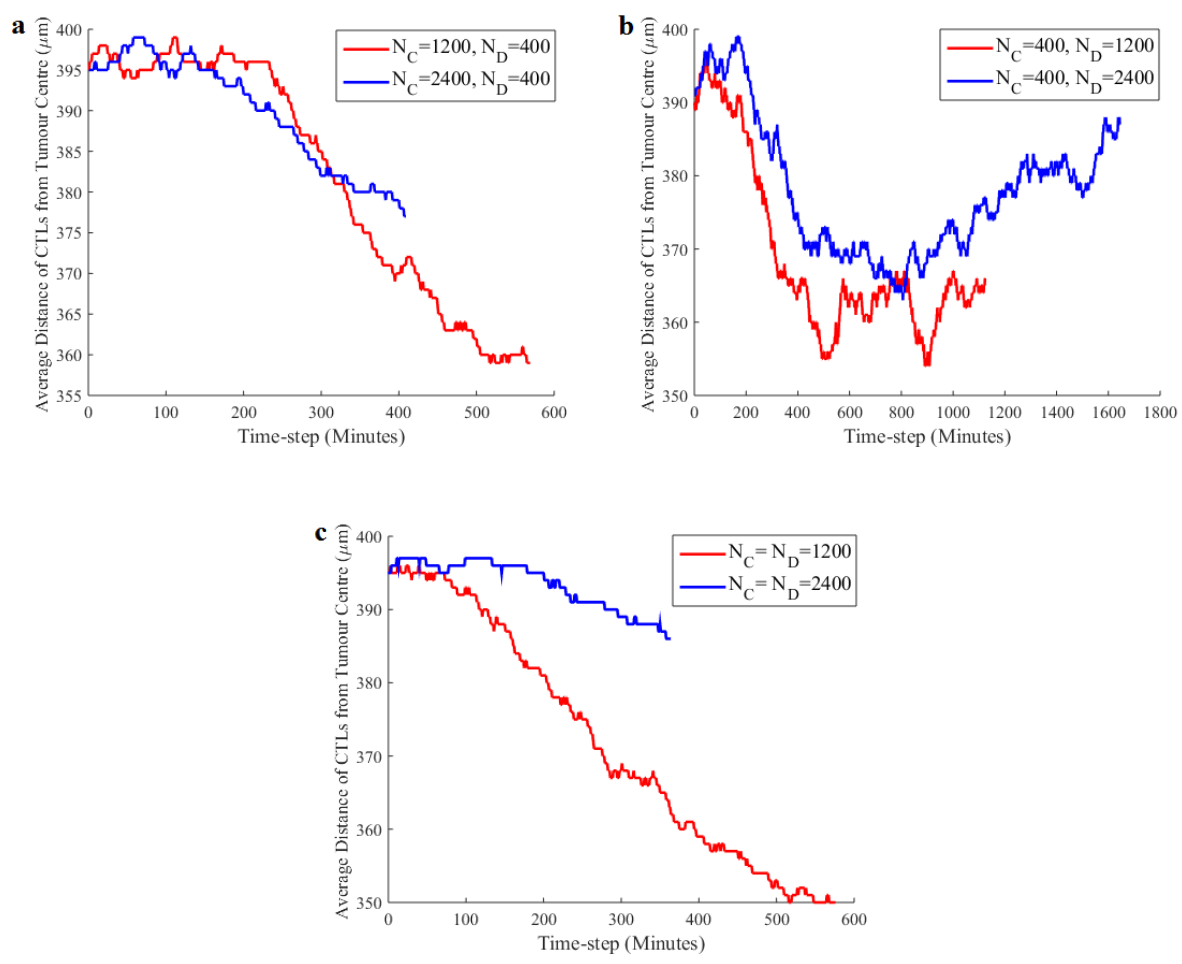

Fig. 5 Increasing the number of DCs can cause overcrowding. Samples of the time evolution of the average distance of the CTLs from the centre of the tumour for different numbers of CTLs, $N_{C}$, and DCs, $N_{D}$. The simulations are run and the average distance of the CTLs from the tumour centre is calculated until all tumour cells are removed from the system. (a) Increasing the number of CTLs only from 1200 cells (red line) to 2400 cells (blue line) the CTLs do not get as close to the tumour centre; this limits the CTLs' ability to remove the tumour cells. (b) Increasing the number of DCs only from 1200 cells (red line) to 2400 cells (blue line) the CTLs do not get as close to the tumour centre and DCs appear to cause overcrowding, which pushes activated CTLs away from the tumour (see also Online Resources 5-6). (c) Increasing both the number of CTLs and DCs from 1200 cells (red line) to 2400 cells (blue line) we observe that, on average, the CTLs are not as close to the tumour centre, limiting their ability to remove the tumour cells.

3.5 Increasing the activation rates of DCs and CTLs has little effect on tumour removal

Antigen presentation has been shown to be important in tumour removal (Boissonnas et al, 2007). Hence, we investigate whether altering the rate at which DCs become activated by the tumour antigen, $D_{A c t}$, and the rate at which CTLs are activated by DCs, $C_{A c t}$, has an effect on tumour removal time. IBM simulations were again run over a $72 \mathrm{hr}$ period or until all of the tumour cells were removed from the system. All parameters remained at set values from Table 2 except for $D_{A c t}$ and $C_{A c t}$. We consider two tumour situations: a smaller tumour made up of 400 cells at the beginning of simulations, Fig.7, and a larger tumour made up of 1200 tumour cells at the beginning of simulations, Fig.8. Simulations were run 

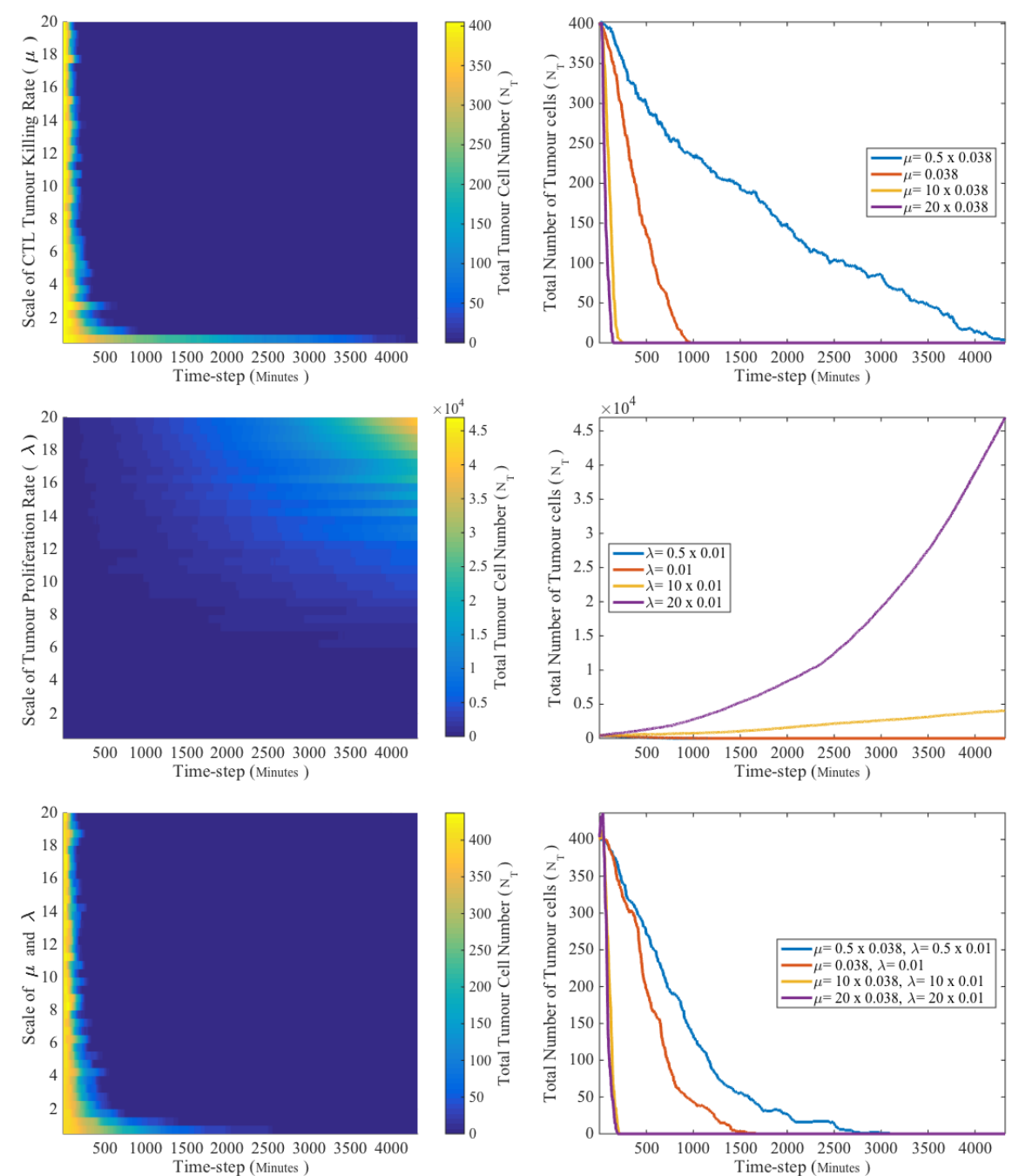

Fig. 6 The ratio between the killing rate of tumour cells by CTLs and the tumour cell proliferation rate is a crucial parameter in tumour removal. Heat maps showing the evolution of the number of tumour cells over time for 40 different values of the rate at which CTLs kill tumour cells, $\mu$, and/or the tumour proliferation rate, $\lambda$ (left panels). Sample time evolutions of the tumour cell number for 4 values of $\mu$ and/or $\lambda$ (right panels). In all cases under consideration, at the beginning of simulations the tumour contained 400 cells. Top panels: Increasing $\mu$ we observe a decrease in tumour removal time, with little difference between larger values. Middle panels: Increasing $\lambda$ results in an increase in tumour removal time and eventually a larger number of tumour cells remaining. Bottom panels: Varying both $\lambda$ and $\mu$ at equal ratios results in a decrease in tumour removal time for increasing values of $\mu$ and $\lambda$, although (in general) tumour removal is slower than in the case where only $\mu$ is varied 
with a range of 40 values of $D_{\text {Act }}$ and/or $C_{A c t}$ where both rates were scaled ranging from a half to twenty times the original parameter values found in Table 2. Heat maps were created to show the total number of tumour cells over time for varying values of $D_{A c t}$ and/or $C_{A c t}$, left panels of Fig.7 and left panels of Fig.8. Selected values of a half, one, ten and twenty times the original parameter values were chosen to give a sample of the dynamics in each case, right panels of Fig.7 and right panels of Fig.8. In both the larger and smaller tumour situations, altering either $D_{A c t}$ and/or $C_{A c t}$ has no clear effect on tumour removal time, which can be seen in the fluctuating results displayed by the heat maps. However, for the case of a larger tumour, when consider the sample dynamics we observe that larger immune activation rates can lead to slower tumour removal compared to lower activation rates, right panels of Fig.8. This was not observed in the case of a smaller tumour, right panels of Fig.7.

\section{Discussion and conclusions}

In this paper we have developed an individual-based spatial model of tumourimmune competition focussing on cell-cell interactions between cancer cells, dendritic cells and cytotoxic T lymphocytes. The model explicitly takes into account the difference in movement between inactive and activated immune cells. Our simulation results demonstrate that the strategies we adopted to modelling immune cell motion make it possible to qualitatively reproduce trajectories of CTLs observed in experimental data (Boissonnas et al, 2007). In particular, Brownian motion captures the movement of CTLs in the presence of a tumour antigen, whilst a Lévy walk provides a good representation of CTLs' motion when the antigen is not present.

Our theoretical work recapitulates previous experimental results showing that increasing CTL numbers, to an extent, can have a beneficial effect on the immune response to cancer (Spranger, 2016), whereas higher number of DCs can be detrimental to the anti-cancer immune action (Sato et al, 2004). In fact, we have shown that, for a number of DCs given, increasing the number of CTLs leads to a faster tumour removal time. On the other hand, for a given number of CTLs, sufficiently larger numbers of DCs can bring about slower tumour removal than lower numbers. In agreement with the experimental results presented in ( $\mathrm{Li}$ et al, 2010; Ye et al, 2011) - where the authors showed that cellular overcrowding can hamper immune action - our computational results indicate that higher numbers of DCs can lead to overcrowding and prevent activated CTLs from reaching the tumour, which results in reduced success of tumour removal. This could explain instances of unsuccessful dendritic cell therapy (Ahmed et al, 2014; Butterfield, 2013; Garg et al, 2017; Lim et al, 2007) and suggests that increasing CTL numbers may be a better objective for immunotherapy rather than increasing DC numbers.

We have shown that the tumour cell proliferation rate (i.e. the parameter $\lambda$ ) has a pronounced effect on tumour-immune competition. In fact, for a given value of the rate at which CTLs can kill tumour cells (i.e. the parameter $\mu$ ), larger values of $\lambda$ result in longer tumour removal times, eventually leading to situations of uncontrolled tumour growth. However, if the value of the parameter $\mu$ is also increased, in agreement with previous theoretical works (d'Onofrio and Ciancio, 2011; Frascoli et al, 2014; Matzavinos et al, 2004), our model predicts that tumour 

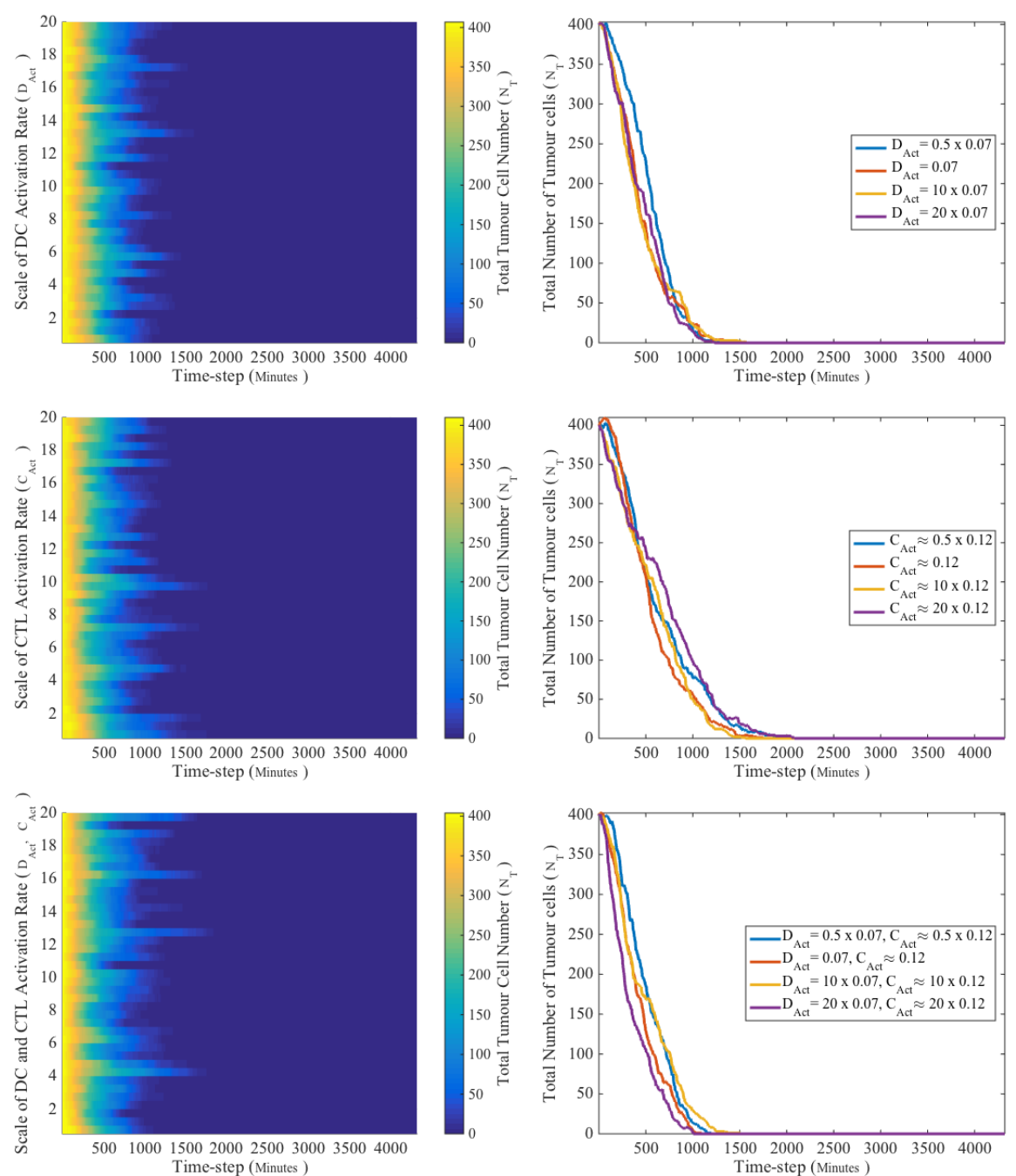

Fig. 7 Increasing the activation rates of DCs and CTLs has little effect on tumour removal (400 tumour cells initially). Heat maps showing the evolution of the number of tumour cells over time for 40 different values of the activation rate of the dendritic cells, $D_{A c t}$, and the activation rate of the cytotoxic $\mathrm{T}$ lymphocytes, $C_{\text {Act }}$ (left panels). Sample time evolutions of the tumour cell number for 4 selected activation rate values (right panels). Top panels: Varying $D_{A c t}$ does not have a great effect on tumour removal time as all values give similar results. Middle panels: Varying $C_{A c t}$ we observe similar results for each value. However, larger values (twenty times the original value) result in slower tumour removal than smaller values (half of the original value). Bottom panels: Varying both $D_{A c t}$ and $C_{A c t}$ we obtain similar results for each value, with a general slight decrease in tumour removal time 

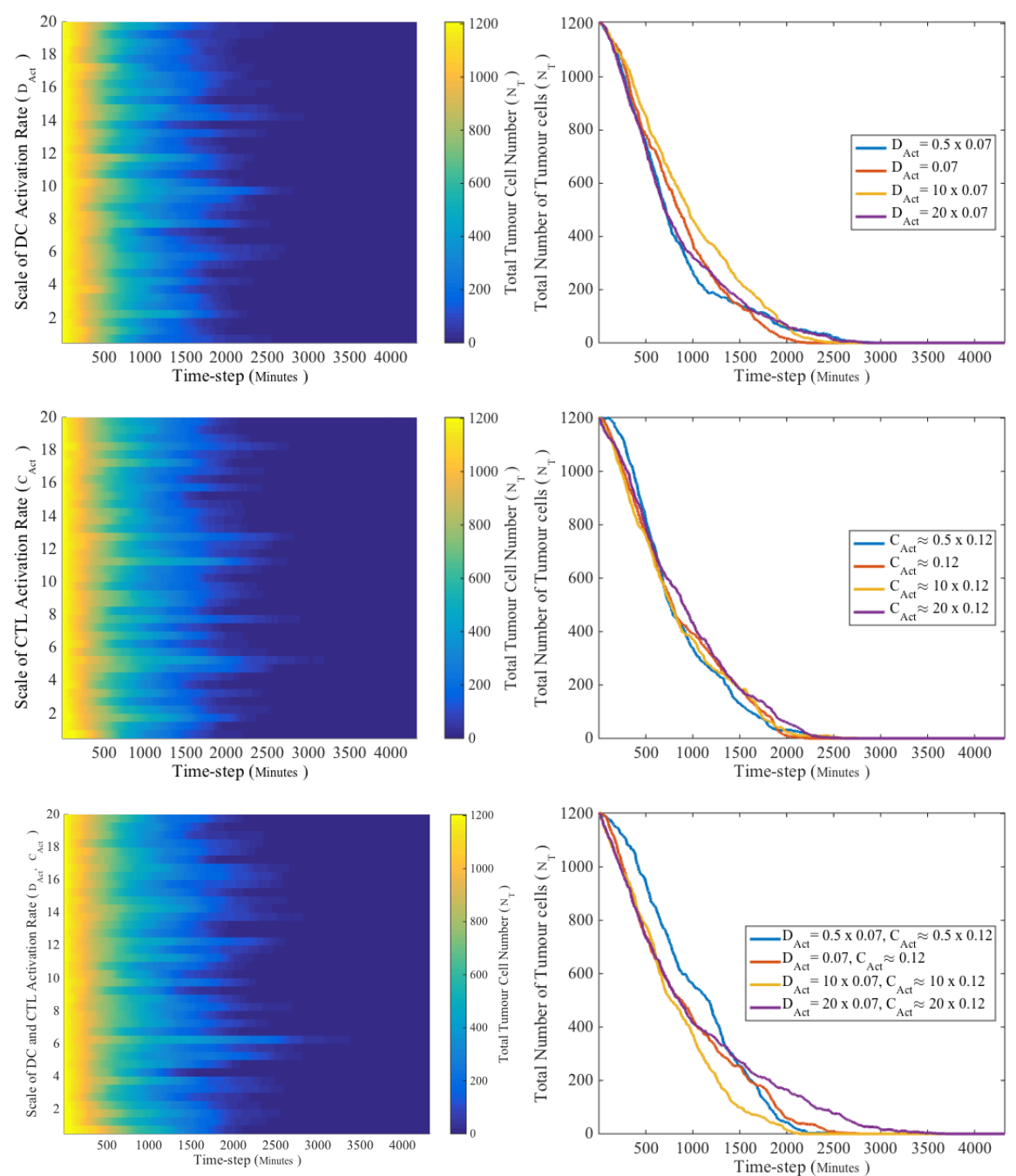

Fig. 8 Increasing the activation rates of DCs and CTLs has little effect on tumour removal (1200 tumour cells initially). Heat maps showing the evolution of the number of tumour cells over time for 40 different values of the activation rate of the dendritic cells, $D_{A c t}$, and the activation rate of the cytotoxic T lymphocytes, $C_{\text {Act }}$ (left panels). Sample time evolutions of the tumour cell number for 4 selected activation rate values (right panels). Top panels: Varying $D_{\text {Act }}$ does not have a great effect on tumour removal time and for all values we obtain similar results. However, larger values (twenty times the original value) result into slower tumour removal than smaller values in the sample dynamics. Middle panels: Varying $C_{A c t}$ we observe similar results for each value. However, larger values (twenty times the original value) result in slower tumour removal than smaller activation rates in the sample dynamics. Bottom panels: Varying both $D_{A c t}$ and $C_{A c t}$ we observe that larger values (twenty times the original value) correspond to slower tumour removal than smaller values 
eradication can be reestablished. Such results illustrate how the ratio $\mu / \lambda$ is a crucial determinant of the outcome of anti-tumour immune action.

The outcomes of our model demonstrate that, in general, increasing the activation rates of CTLs and DCs (i.e. the parameters $C_{A c t}$ and $D_{A c t}$, respectively) has very little effect on removal of the tumour. Taken together, our results testify to the idea that choosing the activation rates of CTLs and DCs as designated targets in adoptive cell therapy, or other forms of immunotherapy, may not enhance the efficacy of the immune response against solid tumours.

We conclude with an outlook on possible extensions of the present work. In our model, immune cell proliferation and decay are not considered for simplicity. It may be interesting to see if there is a change in dynamics upon inclusion of these processes. Exhaustion and deactivation of the immune cells are also not considered due to a lack of relevant data. However, preliminary simulations where DCs could only activate limited numbers of cells gave similar results to the case where there was no restriction on the number of CTLs a DC could activate. Similarly, when CTLs could only kill a limited number of tumour cells, the results obtained were similar to those of the case where there wass no restriction on the number of tumour cells a CTL could kill. This suggests that the general conclusions of our work would remain intact even in the presence of immune cell exhaustion or deactivation.

Tumour antigens can be either tumour specific antigens (TSAs), tumour associated antigens (TAAs) or cancer/testis antigens (CTAs). TSAs are proteins that only tumour cells produce, that is, no normal cells will produce them. TAAs are most common and are produced by tumour cells, but also by some normal cells at a low level of expression. This could impact the immune response to cancer as CTLs may identify non-cancer cells as harmful due to the presence of TAAs, potentially leading to autoimmunity. CTAs are specific to either tumour cells or reproductive cells, but are unlikely to be found elsewhere (Yarchoan et al, 2017). The presence of different types of antigens, or the ability of the tumour to mutate producing different types of antigen, could be studied by extending the model. In the antigen presentation process, major histocompatibility complex (MHC) molecules on the surface of the dendritic cell bind to the antigens that are within the DC and transport them to the external cell surface. Some tumours have been shown to down-regulate MHC molecules preventing this process from occurring. In turn, this blocks the activation of CTLs (Garrido et al, 2010). Our model could be further developed to incorporate such an immunoevasion process. Tumour cells can also directly attack the lymphocytes. Normally Fas ligand (FasL) from the lymphocyte binds to the Fas receptor on the tumour cell surface, they aggregate to form a Fas associated death domain which activates a caspase cascade resulting in apoptosis of the tumour cell (Hersey and Zhang, 2001). However, tumour cells can also produce FasL and activate apoptosis of the CTLs through their Fas receptors (Stewart and Abrams, 2008). This is a further level of complexity which could be incorporated into our model.

\section{References}

Ahmed MD, Bae YS, et al (2014) Dendritic cell-based therapeutic cancer vaccines: past, present and future. Clin Exp Vaccine Res 3(2):113-116 
Al-Tameemi M, Chaplain MAJ, d'Onofrio A (2012) Evasion of tumours from the control of the immune system: Consequences of brief encounters. Biol Direct $7(1): 31-31$

Andersen R, Donia M, Ellebaek E, Borch TH, Kongsted P, Iversen TZ, et al (2016) Long-lasting complete responses in patients with metastatic melanoma after adoptive cell therapy with tumor-infiltrating lymphocytes and an attenuated IL-2 regimen. Clin Cancer Res 22(15):3734-3745

Basu R, Whitlock BM, Husson J, Le Floch A, Jin W, Oyler-Yaniv A, Dotiwala F, Giannone G, Hivroz C, Biais N, et al (2016) Cytotoxic T cells use mechanical force to potentiate target cell killing. Cell 165(1):100-110

Bellomo N, Delitala M (2008) From the mathematical kinetic, and stochastic game theory to modelling mutations, onset, progression and immune competition of cancer cells. Phys Life Rev 5(4):183-206

Bianca C, Chiacchio F, Pappalardo F, Pennisi M (2012) Mathematical modeling of the immune system recognition to mammary carcinoma antigen. BMC Bioinformatics 13(17 Supplement):S21

Boissonnas A, Fetler L, Zeelenberg IS, Hugues S, Amigorena S (2007) In vivo imaging of cytotoxic $\mathrm{T}$ cell infiltration and elimination of a solid tumor. J Exp Med 204(2):345-356

Bunimovich-Mendrazitsky S, Byrne H, Stone L (2008) Mathematical model of pulsed immunotherapy for superficial bladder cancer. Bull Math Biol 70(7):2055-2076

Butterfield LH (2013) Dendritic cells in cancer immunotherapy clinical trials: are we making progress? Front Immunol 4:454

Carreno BM, Magrini V, Becker-Hapak M, Kaabinejadian S, Hundal J, Petti AA, Ly A, Lie WR, Hildebrand WH, Mardis ER, et al (2015) A dendritic cell vaccine increases the breadth and diversity of melanoma neoantigen-specific $\mathrm{T}$ cells. Science 348(6236):803-808

Cattani C, Ciancio A, dOnofrio A (2010) Metamodeling the learning-hiding competition between tumours and the immune system: a kinematic approach. Math Comput Model 52(1):62-69

Chowdhury D, Sahimi M, Stauffer D (1991) A discrete model for immune surveillance, tumor immunity and cancer. J Theor Biol 152(2):263-270

Christophe C, Müller S, Rodrigues M, Petit AE, Cattiaux P, Dupré L, Gadat S, Valitutti S (2015) A biased competition theory of cytotoxic T lymphocyte interaction with tumor nodules. PloS One 10(3):e0120,053

Delitala M, Lorenzi T (2013) Recognition and learning in a mathematical model for immune response against cancer. Discrete Continuous Dyn Syst Ser B 18(4):891914

d'Onofrio A, Ciancio A (2011) Simple biophysical model of tumor evasion from immune system control. Phys Rev E 84(3):031,910

Engelhardt JJ, Boldajipour B, Beemiller P, Pandurangi P, Sorensen C, Werb Z, Egeblad M, Krummel MF (2012) Marginating dendritic cells of the tumor microenvironment cross-present tumor antigens and stably engage tumor-specific T cells. Cancer Cell 21(3):402-417

Frascoli F, Kim PS, Hughes BD, Landman KA (2014) A dynamical model of tumour immunotherapy. Math Biosci 253:50-62

Frigault MJ, Maus MV (2016) Chimeric antigen receptor-modified T cells strike back. Int Immunol p dxw018 
Garg AD, Coulie PG, Van den Eynde BJ, Agostinis P (2017) Integrating nextgeneration dendritic cell vaccines into the current cancer immunotherapy landscape. Trends in Immunology 1392:1-17

Garrido F, Cabrera T, Aptsiauri N (2010) Hard and soft lesions underlying the HLA class I alterations in cancer cells: Implications for immunotherapy. Int J Cancer 127(2):249-256

Goya GF, Marcos-Campos I, Fernandez-Pacheco R, Saez B, Godino J, Asin L, Lambea J, Tabuenca P, Mayordomo JI, Larrad L, et al (2008) Dendritic cell uptake of iron-based magnetic nanoparticles. Cell Biol Int 32(8):1001-1005

Gross G, Eshhar Z (2016) Therapeutic potential of T cell chimeric antigen receptors (CARs) in cancer treatment: Counteracting off-tumor toxicities for safe CAR T cell therapy. Annu Rev Pharmacol Toxicol 56:59-83

Halle S, Keyser KA, Stahl FR, Busche A, Marquardt A, Zheng X, Galla M, Heissmeyer V, Heller K, Boelter J, et al (2016) In vivo killing capacity of cytotoxic $\mathrm{T}$ cells is limited and involves dynamic interactions and $\mathrm{T}$ cell cooperativity. Immunity 44(2):233-245

Hanahan D, Weinberg RA (2011) Hallmarks of cancer: The next generation. Cell 144(5):646-674

Harris TH, Banigan EJ, Christian DA, Konradt C, Wojno EDT, Norose K, Wilson EH, John B, Weninger W, Luster AD, et al (2012) Generalized Lévy walks and the role of chemokines in migration of effector CD8+ T cells. Nature 486(7404):545-548

Hersey P, Zhang X (2001) How melanoma cells evade trail-induced apoptosis. Nat Rev Cancer 1(2):142-150

Hu WY, Zhong WR, Wang FH, Li L, Shao YZ (2012) In silico synergism and antagonism of an anti-tumour system intervened by coupling immunotherapy and chemotherapy: a mathematical modelling approach. Bull Math Biol 74(2):434452

Ikeda H (2016) T-cell adoptive immunotherapy using tumor-infiltrating $\mathrm{T}$ cells and genetically engineered TCR-T cells. Int Immunol p dxw022

Joshi B, Wang X, Banerjee S, Tian H, Matzavinos A, Chaplain MAJ (2009) On immunotherapies and cancer vaccination protocols: A mathematical modelling approach. J Theor Biol 259:820-827

Kolev M (2003) Mathematical modeling of the competition between acquired immunity and cancer. Int J of Appl Math Comput Sci 13:289-296

Kuznetsov VA, Knott GD (2001) Modeling tumor regrowth and immunotherapy. Math Comput Model 33(12):1275-1287

Kuznetsov VA, Makalkin IA, Taylor MA, Perelson AS (1994) Nonlinear dynamics of immunogenic tumors: Parameter estimation and global bifurcation analysis. Bull Math Biol 56(2):295-321

Li X, Yang A, Huang H, Zhang X, Town J, Davis B, Cockcroft DW, Gordon JR (2010) Induction of type $2 \mathrm{~T}$ helper cell allergen tolerance by IL-10-differentiated regulatory dendritic cells. Am J Respir Cell Mol Biol 42(2):190-199

Lim DS, Kim JH, Lee DS, Yoon CH, Bae YS (2007) DC immunotherapy is highly effective for the inhibition of tumor metastasis or recurrence, although it is not efficient for the eradication of established solid tumors. Cancer Immunol Immunother 56(11):1817-1829

Lin Erickson AH, Wise A, Fleming S, Baird M, Lateef Z, Molinaro A, TebohEwungkem M, de Pillis LG (2009) A preliminary mathematical model of skin 
dendritic cell trafficking and induction of $\mathrm{T}$ cell immunity. Discrete Continuous Dyn Syst Ser B 12:323-336

Lorenzi T, Chisholm RH, Melensi M, Lorz A, Delitala M (2015) Mathematical model reveals how regulating the three phases of T-cell response could counteract immune evasion. Immunology 146(2):271-280

Matzavinos A, Chaplain MAJ, Kuznetsov VA (2004) Mathematical modelling of the spatio-temporal response of cytotoxic T-lymphocytes to a solid tumour. Math Med Biol 21(1):1-34

Messerschmidt JL, Prendergast GC, Messerschmidt GL (2016) How cancers escape immune destruction and mechanisms of action for the new significantly active immune therapies: Helping non-immunologists decipher recent advances. Oncologist 21(2):233-243

Modiano JF, Bellgrau D (2016) Fas ligand based immunotherapy: A potent and effective neoadjuvant with checkpoint inhibitor properties, or a systemically toxic promoter of tumor growth? Discov Med 21(114):109-116

Pappalardo F, Musumeci S, Motta S (2008) Modeling immune system control of atherogenesis. Bioinformatics 24(15):1715-1721

Pitt JM, Marabelle A, Eggermont A, Soria JC, Kroemer G, Zitvogel L (2016) Targeting the tumor microenvironment: Removing obstruction to anticancer immune responses and immunotherapy. Ann Oncol 8:1482-1492

Prue RL, Vari F, Radford KJ, Tong H, Hardy MY, DRozario R, Waterhouse NJ, Rossetti T, Coleman R, Tracey C, et al (2015) A phase I clinical trial of CD1c (BDCA-1)+ dendritic cells pulsed with HLA-A* 0201 peptides for immunotherapy of metastatic hormone refractory prostate cancer. J Immunother $38(2): 71-76$

Rozenberg G (2011) Microscopic Haematology: A Practical Guide for the Laboratory, 3rd edn, Elsevier Australia, chap B4:Lymphocytes, p 106

Sato T, Terai M, Yasuda R, Watanabe R, Berd D, Mastrangelo MJ, Hasumi K (2004) Combination of monocyte-derived dendritic cells and activated T cells which express CD40 ligand: a new approach to cancer immunotherapy. Cancer Immunol Immunother 53(1):53-61

Schreibelt G, Bol KF, Westdorp H, Wimmers F, Aarntzen EHJG, Duivemande Boer T, van de Rakt MWMM, Scharenborg NM, de Boer AJ, Pots JM, et al (2016) Effective clinical responses in metastatic melanoma patients after vaccination with primary myeloid dendritic cells. Clin Cancer Res 22(9):21552166

Shay JW, Zou Y, Hiyama E, Wright WE (2001) Telomerase and cancer. Hum Mol Gen 10(7):677-685

Spranger S (2016) Mechanisms of tumor escape in the context of the T-cellinflamed and the non-T-cell-inflamed tumor microenvironment. Int Immunol $\mathrm{p}$ dxw014

Stewart TJ, Abrams SI (2008) How tumours escape mass destruction. Oncogene 27(45):5894-5903

Takayanagi T, Ohuchi A (2001) A mathematical analysis of the interactions between immunogenic tumor cells and cytotoxic $\mathrm{T}$ lymphocytes. Microbiol Immunol 45(10):709-715

Tan MP, Gerry AB, Brewer JE, Melchiori L, Bridgeman JS, Bennett AD, Pumphrey NJ, Jakobsen BK, Price DA, Ladell K, et al (2015) T cell receptor binding affinity governs the functional profile of cancer-specific CD8 $+\mathrm{T}$ 
cells. Clin Exp Immunol 180(2):255-270

Tel J, Aarntzen EHJG, Baba T, Schreibelt G, Schulte BM, Benitez-Ribas D, Boerman OC, Croockewit S, Oyen WJG, van Rossum M, et al (2013) Natural human plasmacytoid dendritic cells induce antigen-specific T-cell responses in melanoma patients. Cancer Res 73(3):1063-1075

Weinberg RA (2007a) Crowd control: Tumour immunology and immunotherapy. In: Weinberg RA (ed) The Biology of Cancer, Garland Science, U.S.A, chap 15, pp $655-724$

Weinberg RA (2007b) Moving out: Invasion and metastasis. In: Weinberg RA (ed) The Biology of Cancer, Garland Science, U.S.A, chap 14, pp 587-654

Wilgenhof S, Van Nuffel AMT, Corthals J, Heirman C, Tuyaerts S, Benteyn D, De Coninck A, Van Riet I, Verfaillie G, Vandeloo J, et al (2011) Therapeutic vaccination with an autologous mRNA electroporated dendritic cell vaccine in patients with advanced melanoma. J Immunother 34(5):448-456

Wilgenhof S, Corthals J, Heirman C, van Baren N, Lucas S, Kvistborg P, Thielemans K, Neyns B (2016) Phase II study of autologous monocyte-derived mRNAelectroporated dendritic cells (TriMixDC-MEL) plus ipilimumab in patients with pretreated advanced melanoma. J Clin Oncol 34(12):1330-1338

Wilkie KP, Hahnfeldt P (2013) Mathematical models of immune-induced cancer dormancy and the emergence of immune evasion. Interface Focus 3(4):20130,010

Yarchoan M, Johnson BA, Lutz ER, Laheru DA, Jaffee EM (2017) Targeting neoantigens to augment antitumour immunity. Nat Rev Cancer 17(1):209-222

Ye Q, Loisiou M, Levine BL, Suhoski MM, Riley JL, June CH, Coukos G, Powell DJ (2011) Engineered artificial antigen presenting cells facilitate direct and efficient expansion of tumor infiltrating lymphocytes. J Transl Med 9(1):131

\section{Appendix}

Table 2 Values of parameters used in the model

\begin{tabular}{llll}
\hline Symbol & Description & Value/ Units & References \\
\hline$\Delta_{t}$ & time-step of simulations & $1 \mathrm{~min}$ & (Boissonnas et al, 2007) \\
$\Delta_{x(y)}$ & grid spacing in the x ( y) direction & $10 \mu \mathrm{m}$ & Calculated \\
$N_{T}$ & total number of tumour cells & initial values: 400 or $1200 \mathrm{cells}$ & (Christophe et al, 2015) \\
$N_{C}$ & total number of CTLs & $400 \mathrm{cells}$ & (Christophe et al, 2015) \\
$N_{D}$ & total number of DCs & $400 \mathrm{cells}$ & Estimated \\
$\mu$ & rate at which a CTL kills a tumour cell & $0.03 \mathrm{cells} \mathrm{min}^{-1}$ & (Christophe et al, 2015) \\
$\lambda$ & tumour cell proliferation rate & $0.001 \mathrm{~min}^{-1}$ & (Christophe et al, 2015) \\
$D_{A c t}$ & DC activation rate & $0.07 \mathrm{cells} \mathrm{min}^{-1}$ & (Bianca et al, 2012) \\
$C_{A c t}$ & CTL activation rate & $\approx 0.12 \mathrm{cells}^{-1} \mathrm{~min}^{-1}$ & (Engelhardt et al, 2012) \\
$\alpha$ & Lévy walk exponent & 1.15 & (Harris et al, 2012) \\
\hline
\end{tabular}



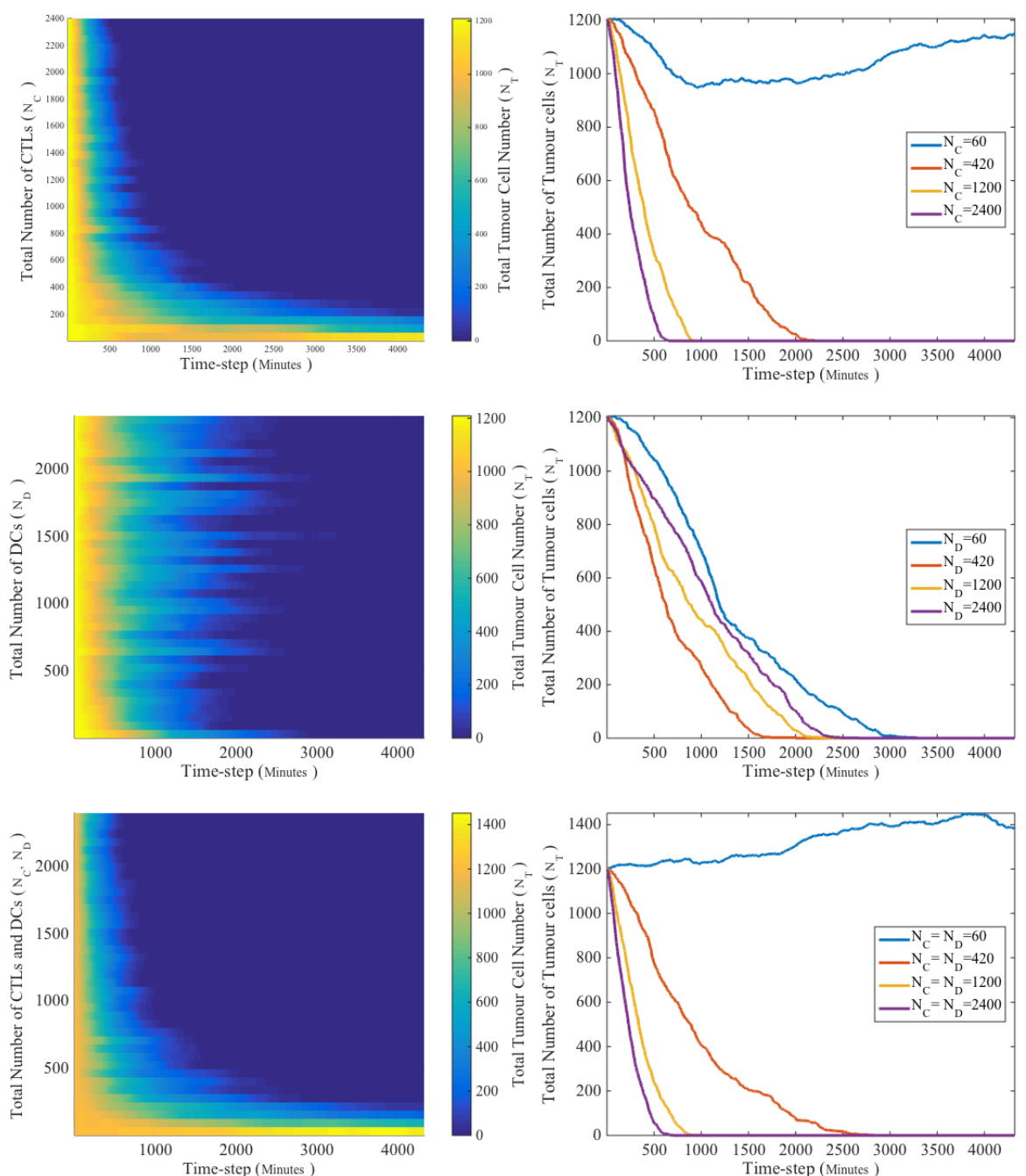

Fig. 9 Increasing the number of DCs can lead to longer tumour removal times. Heat maps showing the time evolution of the number of tumour cells for 40 different numbers of immune cells (left panels) and selected samples of the time evolution of the number of tumour cells for 4 different numbers of immune cells (right panels). In all cases under consideration, at the beginning of simulations the tumour contained 1200 cells. Top panels: Increasing the CTL number $N_{C}$ we observe a general decrease in tumour removal time. Middle panels: Increasing the DC number $N_{D}$ above a certain threshold leads to longer tumour removal time. Bottom panels: Increasing both $N_{C}$ and $N_{D}$ causes a decrease in tumour removal time 

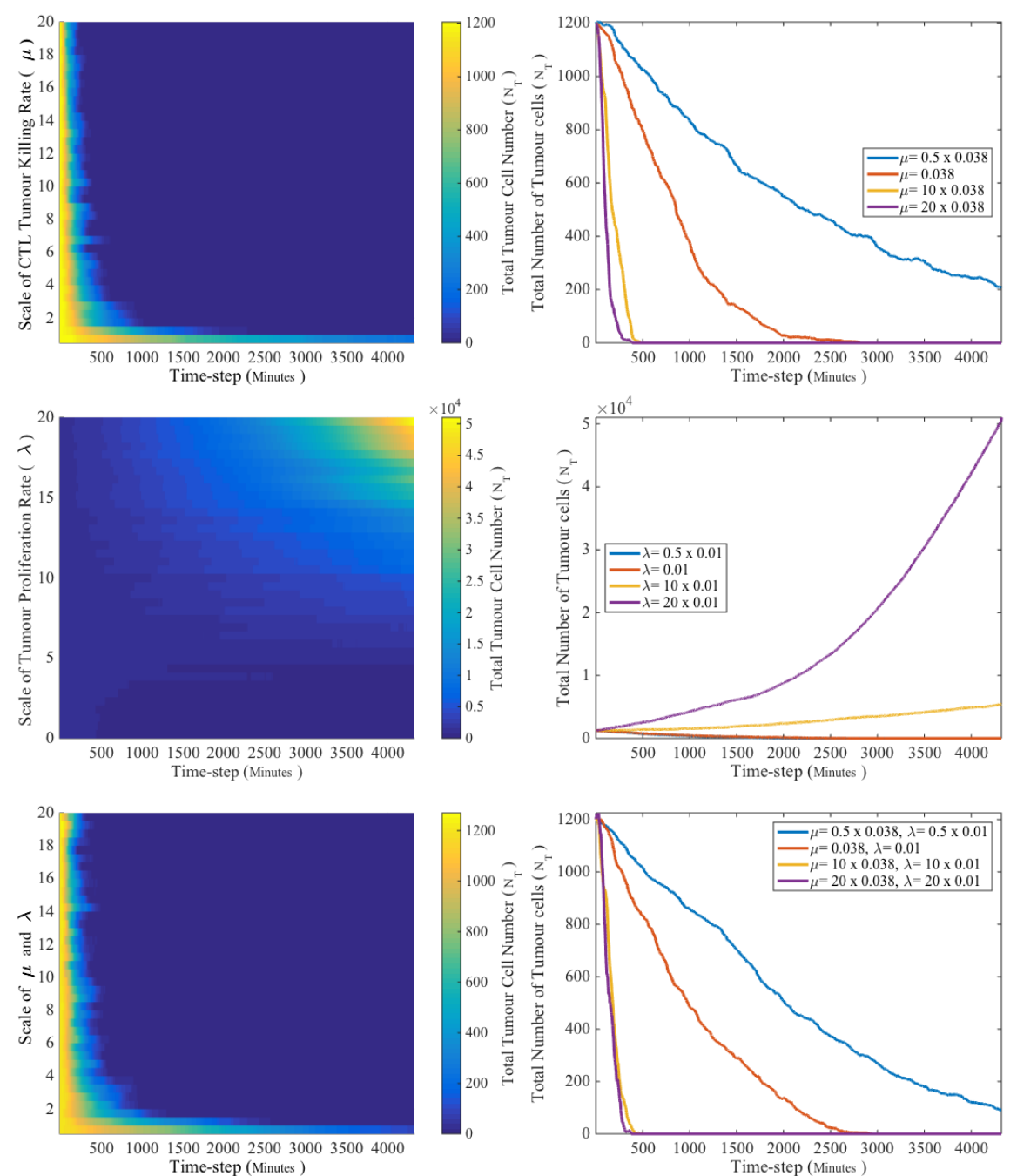

Fig. 10 The ratio between the killing rate of tumour cells by CTLs and the tumour cell proliferation rate is a crucial parameter in tumour removal. Heat maps showing the evolution of the total number of tumour cells over time for 40 different values of the rate at which CTLs kill tumour cells, $\mu$, and/or the tumour cell proliferation rate, $\lambda$ (left panels). Sample time evolutions of the tumour cell number for 4 values of $\mu$ and/or $\lambda$ (right panels). In all cases under consideration, at the beginning of simulations the tumour contained 1200 cells. Top panels: Varying $\mu$ we observe a decrease in tumour removal time with increasing values of $\mu$, with little difference between the larger values. Middle panels: Varying $\lambda$ results in an increase in tumour removal time with increasing $\lambda$ and eventually a larger number of tumour cells remaining. Bottom panels: Varying both $\lambda$ and $\mu$ at equal ratios results in a decrease in tumour removal time for increasing values of $\mu$ and $\lambda$, although (in general) is slower than the case where only $\mu$ is altered 